\title{
An exact expression for photon polarization in Kerr geometry
}

\author{
Anusar Farooqui, Niky Kamran and Prakash Panangaden
}

\begin{abstract}
We analyze the transformation of the polarization of a photon propagating along an arbitrary null geodesic in Kerr geometry. The motivation comes from the problem of an observer trying to communicate quantum information to another observer in Kerr spacetime by transmitting polarized photons. It is essential that the observers understand the relationship between their frames of reference and also know how the photon's polarization transforms as it travels through Kerr spacetime. Existing methods to calculate the rotation of the photon polarization (Faraday rotation) depend on choices of coordinate systems, are algebraically complex and yield results only in the weak-field limit.

We give a closed-form expression for a parallel propagated frame along an arbitrary null geodesic using Killing-Yano theory, and thereby solve the problem of parallel transport of the polarization vector in an intrinsic, geometrically-motivated fashion. The symmetries of Kerr geometry are utilized to obtain a remarkably compact expression for the geometrically induced phase of the photon's polarization. We show that this phase vanishes on the equatorial plane and the axis of symmetry.
\end{abstract}

1 Introduction

2 Kerr geometry

3 Parallel-propagated frame along null geodesics

4 Defining and measuring Faraday rotation

5 Exact, closed form expression for the Faraday rotation in Kerr geometry 


\section{References}

\section{Introduction}

In protocols for quantum communication [19] most of the attention has been focussed on quantum effects such as the problem of coping with noise in the communication mechanism or preserving entanglement. It is typically taken for granted that the participants in the protocol share a frame of reference. However, a closer analysis by Bartlett et al. [2,3] has revealed the importance of sharing a frame. They have even quantified the degree to which a partially shared frame constitutes shared information. The present paper is motivated by these considerations, however, we do not address the quantum information-theoretic issues which would involve a study of the evolution of the quantum state.

Instead, we isolate the classical geometric aspects and study them in Kerr geometry. Specifically, we study how two participants in a quantum communication protocol involving transmission of polarized photons - henceforth we will call them observers - could share a frame in Kerr geometry and how the polarization of a linearly polarized photon would transform as it travels from one observer to the other. It is crucial that this transformation reflect what would be seen by the observers. Furthermore, the quantity we report should be intrinsic to the geometry of the spacetime and not correspond to some arbitrarily chosen coordinate system or frame.

We have two observers called Alice and Bob. Alice sends a linearly polarized photon to Bob; she has chosen the polarization vector to be at some angle in with respect to some axes which she has chosen in the plane of polarization of the photon, which is a 2-plane orthogonal to the direction of propagation of the photon. Bob receives this photon which has travelled through the Kerr spacetime to reach him. In order for Bob to measure the polarization of the photon and know what angle Alice intended to communicate to him, he needs to know how their frames correspond and how the photon polarization has been transformed by the background geometry. 
In Minkowski geometry, the problem is straightforward. Since, the background geometry does not affect the polarization of the photon, one only needs to solve the problem of sharing frames. For a pair of observers who start at the same event with a known relation between their frames, one can Fermi-Walker transport their frames to determine how their frames relate at the point where photon transmission occurs.

At this level of generality the problem is intractable in the Kerr geometry since the Fermi-Walker transport of frames along general timelike curves in Kerr geometry is still an open problem. Note that we are not interested in obtaining reference frames per se. What is required for two observers to exchange quantum information using polarized photons is shared knowledge of basis vectors in which the measurement is performed.

Thus, we seek an intrinsic, geometrically defined measurement basis along specific trajectories. We show how Kerr geometry allows for such a protocol; one which simultaneously solves the problem of sharing frames and minimizes the informational requirement on the observers.

The gravitationally induced rotation of the polarization vector in Kerr geometry has been investigated in the weak field limit by [23],[21],[15],[10], [16], [20],[22]. Extant methods rely on the existence of the Walker-Penrose conserved quantity to solve the problem of parallel propagating the polarization vector along a null geodesic [8]. The estimates are difficult to reconcile because they do not take into account the role of reference frames. However, there is a virtual consensus that the acquired phase is zero in Schwarzschild geometry.

More recently, Brodutch, Demarie and Terno [4] have chosen observers equipped with an orthonormal frame, located at fixed values of $(r, \vartheta, \varphi)$ in Boyer-Lindquist coordinates. They make a physically motivated choice of basis vectors for the plane of polarization by requiring that the acquired phase be zero in the Schwarzschild limit. They find that the acquired phase on the equatorial place is zero and argue that this is because motion on the equatorial plane in Kerr geometry is qualitatively similar to Schwarzschild spacetime. Though their results only hold in the weak field limit, their paper represents a significant advance in the field.

We take a geometric approach. First, our choice of observers is motivated by the intrinsic geometry of Kerr spacetime. We choose a class of observers whose 4-velocities are symmetric linear combinations of the principal null directions of the Weyl tensor. We show how this class of observers is uniquely suited to analyze the behaviour of test particles near the horizon. Second, we endow these observers with a symmetric frame by exploiting the existence of the involutive isometry obtained by simultaneous time- and rotation-reversal 
of the Kerr black hole. This greatly simplifies our expressions. Third, we use the existence of the principal null directions and other special features of Kerr geometry to fix the definition of the plane of polarization and of the measurement basis. This measurement protocol is allowed by the specific symmetry structure of Kerr geometry. It is simply unavailable in Minkowski spacetime where no direction is similarly privileged. Fourth, we use KillingYano theory to construct a parallel propagated frame along the null geodesic (a problem first solved by Marck [18] in a related but slightly different form), thereby reducing the transport problem to one of raising and lowering frame indices. This allows us to obtain a remarkably compact exact expression for Faraday rotation everywhere in the zone of outer communication in Kerr spacetime.

We proceed as follows. Section 2 lays out the geometry and symmetries of the Kerr solution, as well as describes the null geodesic equations. The construction of the parallel propagated frame is given in Section 3. In section 4, we set out our choice of observers and the measurement protocol. We then prove that there is no Faraday rotation for photons confined to the equatorial plane and the axis of symmetry. We show how this immediately implies the vanishing of the acquired phase in Schwarzschild spacetime as well. Section 5 gives the derivation of the closed form expression for the Faraday rotation. In Section 6, we discuss the plots of a few null geodesics (provided in Appendix B) and their associated Faraday rotation. We conclude with some remarks about the physical significance of the results and some possible avenues for future work.

\section{Kerr geometry}

Our goal in this section is to recall some of the salient geometric properties of the Kerr metric that will be used to calculate the Faraday rotation undergone by the polarization vector of a photon. (Throughout this paper, a photon will be thought of as a classical zero rest mass particle moving along an affinely parametrized null geodesic.) We shall see that the remarkable symmetry and separability properties of Kerr geometry make it possible to obtain an exact expression for the Faraday rotation, which we will derive in Section 5 and will subsequently interpret geometrically.

We begin by recalling that the Kerr metric is a two-parameter family of solutions of the Einstein vacuum equations defined on the manifold $M \equiv \mathbb{R}^{2} \times S^{2}$ and describing the outer geometry of a rotating black hole in equilibrium. In Boyer-Lindquist coordinates $\left(x^{i}\right)=(t, r, \vartheta, \varphi)$ with $-\infty<$ $t<+\infty, r_{+}<r<+\infty, 0 \leq \vartheta \leq \pi, 0 \leq \varphi<2 \pi$, the Kerr metric takes the 
form

$d s^{2}=\frac{\Delta}{\Sigma}\left(d t-a \sin ^{2} \vartheta d \varphi\right)^{2}-\frac{\Sigma}{\Delta} d r^{2}-\Sigma d \vartheta^{2}-\frac{\sin ^{2} \vartheta}{\Sigma}\left(a d t-\left(r^{2}+a^{2}\right) d \varphi\right)^{2}$

with

$$
\Sigma(r, \vartheta)=r^{2}+a^{2} \cos ^{2} \vartheta, \quad \Delta(r)=r^{2}-2 M r+a^{2}
$$

The parameters $M>0$ and $a \geq 0$ labeling the solutions within the Kerr family correspond respectively to the mass and angular momentum per unit mass of the black hole, as measured from infinity. We shall restrict our attention to the non-extreme case $M>a \geq 0$, in which case the function $\Delta(r)$ has two distinct zeros,

$$
r_{ \pm}=M \pm \sqrt{M^{2}-a^{2}}
$$

with $r_{+}$corresponding to the lower limit of the range of the Boyer-Lindquist coordinate $r$. It is well-known that the Kerr metric can be analytically continued across the hypersurfaces $r=r_{+}$and $r=r_{-}$in such a way that these become null hypersurfaces in the extended manifold, corresponding respectively to the event and Cauchy horizons of the Kerr black hole geometry. We shall however be interested in the region $r>r_{+}$, which describes the space-time geometry outside the event horizon of a Kerr black hole with parameters $M$ and $a$.

The Weyl conformal curvature tensor of the Kerr solution is of Petrov type D, meaning that it admits a pair of repeated principal null directions, each of which is defined up to multiplication by a non-zero scalar function. These repeated principal null directions give rise to null congruences which are geodesic and shear-free as a consequence of the Goldberg-Sachs Theorem. We choose the scale factors in such a way that the principal null directions are given by

$$
\ell=\ell^{i} \frac{\partial}{\partial x^{i}}=\frac{1}{\sqrt{2 \Sigma \Delta}}\left(\left(r^{2}+a^{2}\right) \frac{\partial}{\partial t}+\sqrt{\Delta} \frac{\partial}{\partial r}+a \frac{\partial}{\partial \varphi}\right)
$$

and

$$
\boldsymbol{n}=n^{i} \frac{\partial}{\partial x^{i}}=\frac{1}{\sqrt{2 \Sigma \Delta}}\left(\left(r^{2}+a^{2}\right) \frac{\partial}{\partial t}-\sqrt{\Delta} \frac{\partial}{\partial r}+a \frac{\partial}{\partial \varphi}\right)
$$


The choice of scale factors leading to (2.3) and (2.4) will be characterized geometrically through an involutive isometry admitted by the Kerr metric (see (2.12)). In particular, the vector fields (2.3) and (2.4), which form part of the symmetric null frame constructed by Debever et al. [9], will play an important in the geometrical characterization of the class of observers that we shall consider in our calculation of the Faraday rotation.

The Kerr metric enjoys remarkable symmetry properties which we will exploit systematically in our calculation of the Faraday rotation and which we now summarize.

First of all, the Kerr metric admits a two-parameter Abelian isometry group that acts orthogonally transitively on time-like orbits, meaning that the orbits of the group action are time-like 2-surfaces with the property that the distribution of 2-planes orthogonal to the orbits is integrable. The orthogonal transitivity is manifest in the Boyer-Lindquist coordinates since the metric does not admit cross terms mixing the differentials $d r$, $d \vartheta$ with the differentials $d t, d \varphi$. In Boyer-Lindquist coordinates, the action of the continuous part of the isometry group is generated by the flows of the pair of commuting Killing vector fields $\partial_{t}$ and $\partial_{\varphi}$, and thus given by

$$
(t, r, \vartheta, \varphi) \mapsto\left(t+c_{1}, r, \vartheta, \varphi+c_{2}\right),
$$

where $c_{1}, c_{2}$ are arbitrary real constants. Furthermore, the isometry group of the Kerr metric admits a discrete subgroup isomorphic to $\mathbb{Z}_{2}$, whose action is not of the form (2.5). More precisely, we say following Carter's terminology [7] that the isometry group is invertible, meaning that at every $x \in M$, there exists a $(1,1)$-tensor $L_{x} \in \operatorname{End}\left(T_{x} M\right)$, which acts as an involutive isometry of $\left(T_{x} M, g_{x}\right)$ and is such that if $\mathcal{O}_{x}$ denotes the orbit of the isometry group through $x$, then

$$
\left.L_{x}\right|_{\left(T_{x} \mathcal{O}_{x}\right)^{\perp}}=\operatorname{id}_{\left(T_{x} \mathcal{O}_{x}\right)^{\perp}},
$$

and for all $X_{x} \in\left(T_{x} \mathcal{O}_{x}\right)^{\perp}$,

$$
L_{x}\left(X_{x}\right)=-X_{x} .
$$

We remark that from a result of Carter [7], it is known that if an isometry group acts orthogonally transitively on non-null orbits then the action is necessarily invertible. ${ }^{1}$ In Boyer-Lindquist coordinates, the involution is

\footnotetext{
${ }^{1}$ This result is not true if the orbits of the isometry group are null
} 
given by

$$
L_{x}=\left.f_{*}\right|_{x}
$$

where $f$ is the isometry given by

$$
(t, r, \vartheta, \varphi) \mapsto(-t, r, \vartheta,-\varphi)
$$

We will commit an abuse of notation and denote both $L_{x}=\left.f_{*}\right|_{x}$ and the dual map $\left.f^{*}\right|_{x}$ by $L$. The involution $L$ will play a key role in defining invariantly the class of observers and frames for which the Faraday rotation will be computed.

We shall work in a Newman-Penrose null coframe

$$
\vartheta^{1}=n_{i} d x^{i}, \vartheta^{2}=\ell_{i} d x^{i}, \vartheta^{3}=-\bar{m}_{i} d x^{i}, \vartheta^{4}=-m_{i} d x^{i}
$$

in which the Kerr metric takes the form

$$
d s^{2}=2\left(\vartheta^{1} \vartheta^{2}-\vartheta^{3} \vartheta^{4}\right) .
$$

Following the construction of Debever et al. [9], this coframe is chosen such that

$$
L \vartheta^{1}=-\vartheta^{2}, L \vartheta^{2}=-\vartheta^{1}, L \vartheta^{3}=-\vartheta^{4}, L \vartheta^{4}=-\vartheta^{3}
$$

Following [9], we refer to this frame as the symmetric coframe. Note that this last requirement eliminates the scaling freedom we would have otherwise had in defining a null coframe adapted to the principal null directions of the Weyl tensor. The corresponding orthonormal symmetric coframe $\left(\boldsymbol{\omega}^{0}, \boldsymbol{\omega}^{1}, \boldsymbol{\omega}^{2}, \boldsymbol{\omega}^{3}\right)$ is then defined by

$$
\begin{aligned}
\boldsymbol{\omega}^{0} & =\frac{1}{\sqrt{2}}\left(\vartheta^{1}+\vartheta^{2}\right), \quad \boldsymbol{\omega}^{1}=\frac{1}{\sqrt{2}}\left(\vartheta^{2}-\vartheta^{1}\right), \\
\boldsymbol{\omega}^{2}=-\frac{1}{\sqrt{2}}\left(\vartheta^{3}+\vartheta^{4}\right), & \boldsymbol{\omega}^{3}=\frac{1}{\sqrt{2}}\left(\vartheta^{3}-\vartheta^{4}\right),
\end{aligned}
$$

and given in Boyer-Lindquist coordinates by 


$$
\begin{aligned}
\boldsymbol{\omega}^{0} & =\sqrt{\frac{\Delta}{\Sigma}}\left(d t-a \sin ^{2} \vartheta d \varphi\right), \\
\boldsymbol{\omega}^{1} & =\sqrt{\frac{\Sigma}{\Delta}} d r, \\
\boldsymbol{\omega}^{2} & =\frac{\sin \vartheta}{\sqrt{\Sigma}}\left(a d t-\left(r^{2}+a^{2}\right) d \varphi\right), \\
\boldsymbol{\omega}^{3} & =\sqrt{\Sigma} d \vartheta .
\end{aligned}
$$

Throughout this paper, we shall reserve lower case Latin indices $a, b, c, \ldots$ to denote components with respect to the orthonormal symmetric coframe (2.14)-(2.17) and the orthonormal frame dual to it. We shall denote the flat spacetime metric used to raise and lower these orthonormal frame indices by $\eta$, where

$$
\eta_{a b}=\eta^{a b}=\left[\begin{array}{llll}
1 & & & \\
& -1 & & \\
& & -1 & \\
& & & -1
\end{array}\right] .
$$

It is well known that in addition to its two-parameter Abelian group of isometries, the Kerr metric posesses further symmetries whose presence is closely tied to the fact that all the known massless and massive wave equations are separable in Boyer-Lindquist coordinates and, where applicable, the symmetric frame. The geometric object that generates all these additional symmetries is a rank two Killing-Yano tensor, that is, a $(0,2)$ skew-symmetric tensor $\left(f_{i j}\right)$ satisfying the Killing-Yano equation

$$
\nabla_{i} f_{j k}+\nabla_{j} f_{i k}=0 .
$$

In Boyer-Lindquist coordinates and in the symmetric orthonormal coframe, any rank 2 Killing-Yano tensor is a constant multiple of

$$
\boldsymbol{f}:=\frac{1}{2} f_{i j} d x^{i} \wedge d x^{j}=-a \cos \vartheta \boldsymbol{\omega}^{0} \wedge \boldsymbol{\omega}^{1}+r \boldsymbol{\omega}^{2} \wedge \boldsymbol{\omega}^{3}
$$

The role played by this Killing-Yano tensor in the separability properties of the Kerr metric stems from the fact that it appears as a "square root" of the quadratic first integral discovered by Carter in his proof of the separability in Kerr geometry of the Hamilton-Jacobi equation for geodesics and the KleinGordon equation for massive scalar fields [6]. More precisely, the symmetric 
$(0,2)$-tensor $\left(K_{i j}\right)$ defined by

$$
K_{i j}=f_{i k} f_{j}^{k}
$$

satisfies the Killing equation

$$
\nabla_{i} K_{j k}+\nabla_{j} K_{k i}+\nabla_{k} K_{i j}=0
$$

and therefore gives rise to a quadratic first integral for the geodesic flow in Kerr geometry first discovered by Carter [5]

$$
\kappa=K^{i j} p_{i} p_{j}
$$

This quadratic first integral exists in addition to the two linear first integrals arising from the presence of the two commuting Killing vector fields $\partial_{t}$ and $\partial_{\varphi}$ and therefore reduces the integration of the geodesic flow to quadratures. For the purposes of calculating the Faraday rotation of a photon, we shall be interested in affinely parametrized null geodesics, for which the equations can be written in first-order form as

$$
\begin{aligned}
\dot{r} & = \pm \frac{\sqrt{R}}{\Sigma} \\
\dot{\vartheta} & = \pm \frac{\sqrt{\Theta}}{\Sigma} \\
\Sigma \Delta \dot{t} & =E\left(\left(r^{2}+a^{2}\right)^{2}-\Delta a^{2} \sin ^{2} \vartheta\right)-2 M r a \Phi, \\
\Sigma \Delta \dot{\varphi} & =2 M r a E+(\Sigma-2 M r) \Phi / \sin ^{2} \vartheta .
\end{aligned}
$$

In these equations, the dot denotes the derivative with respect to an affine parameter $s$, the constant $E$ is the conserved momentum $p_{t}$ corresponding to the energy of zero rest-mass particle moving along the null geodesic, $\Phi$ is the conserved angular momentum $-p_{\varphi}$ along the axis of symmetry of the Kerr black hole, $\kappa$ is Carter's fourth integral of motion given by (2.23), and

$$
\begin{aligned}
& R(r):=\mathbb{P}^{2}-\Delta \kappa, \\
& \Theta(\vartheta):=\kappa-\mathbb{D}^{2},
\end{aligned}
$$

where

$$
\begin{aligned}
\mathbb{P}(r) & :=E\left(r^{2}+a^{2}\right)-a \Phi, \\
\mathbb{D}(\vartheta) & :=a \sin \vartheta E-\Phi / \sin \vartheta .
\end{aligned}
$$




\section{Parallel-propagated frame along null geodesics}

By definition, the polarization 4-vector $\digamma$ of a photon is a vector field along an affinely parametrized null geodesic $\gamma$ with tangent vector $\boldsymbol{K}$ that is both parallel propagated along $\gamma$ and orthogonal to $\boldsymbol{K}$, that is,

$$
\begin{aligned}
& K^{i} \nabla_{i} \digamma^{j}=K^{a} \nabla_{a} \digamma^{b}=0 \\
& K^{i} \digamma^{j} g_{i j}=K^{a} \digamma^{b} \eta_{a b}=0 .
\end{aligned}
$$

In order to solve this transport equations (3.1) and (3.2), we construct a frame that is parallel propagated along an arbitrary null geodesic in Kerr geometry. We shall see that, just as in Marck's original construction [18], the Killing-Yano tensor (2.19) will play a key role.

We first recall that the two commuting Killing vectors admitted by the Kerr metric can be recovered from the Killing-Yano tensor (2.19) using the Hodge duality operator. Indeed, it follows from the defining equation (2.19) for Killing-Yano tensors that the vector fields $\boldsymbol{\xi}$ and $\boldsymbol{\zeta}$ defined by

$$
\xi^{i}:=\frac{1}{3} \nabla_{j} h^{j i}, \quad \zeta^{i}:=-K^{i}{ }_{j} \xi^{j},
$$

where $\left(h_{i j}\right)$ denotes the Hodge dual of $\left(f_{i j}\right)$, are Killing vector fields. Explicitly, with the Killing-Yano tensor $\left(f_{i j}\right)$ given by $(2.20)$, the Hodge dual $\boldsymbol{h}$ is given by

$$
\boldsymbol{h}=\frac{1}{2} h_{i j} d x^{i} \wedge d x^{j}=r \boldsymbol{\omega}^{o} \wedge \boldsymbol{\omega}^{1}+a \cos \vartheta \boldsymbol{\omega}^{2} \wedge \boldsymbol{\omega}^{3},
$$

and we have

$$
\boldsymbol{\xi}=\partial_{t}, \quad \boldsymbol{\zeta}=\partial_{\varphi} .
$$

A parallel propagated frame along the null geodesics of the Kerr metric is now constucted as follows. We follow the construction of Kubiznak et al. [17]. The relevant result is:

Lemma 3.1. Let $\gamma$ be an affinely parametrized null geodesic with tangent vector $\boldsymbol{K}$. Let $\boldsymbol{X}$ be a vector field that is both parallel propagated along $\gamma$

$$
K^{i} \nabla_{i} X^{j}=0,
$$

and orthogonal to $\boldsymbol{K}$,

$$
g_{i j} K^{i} X^{j}=0 .
$$


Then, the vector field $\boldsymbol{Y}$ defined along $\gamma$ by

$$
Y^{i}=X^{j} h_{j}^{i}+\beta_{\boldsymbol{X}} K^{i}
$$

where

$$
\frac{d}{d s} \beta_{\boldsymbol{X}}=g_{k l} X^{k} \xi^{l}
$$

and $\frac{d}{d s}$ denotes differentiation with respect to an affine parameter $s$ along $\gamma$, and $\boldsymbol{\xi}$ is as defined by (3.3), is parallel propagated along $\gamma$.

We now consider an affinely parametrized arbitrary null geodesic $\gamma$ in the Kerr metric and construct a parallel propagated frame along $\gamma$ by repeated application of Lemma 3.1. From now on, we will work exclusively in Carter's symmetric frame, defined as the orthonormal frame dual to the symmetric orthonormal coframe given by (2.14)-(2.17). Vector fields will thus be identified with their components in the symmetric frame and will be represented as four-component row vectors.

Given an affinely parametrized null geodesic $\gamma$, it follows from (2.24)(2.27) that the tangent vector $\boldsymbol{K}=\dot{\gamma}$ is given by

$$
\boldsymbol{K}=\frac{1}{\sqrt{ } \Sigma}\left(\frac{\mathbb{P}}{\sqrt{ } \Delta}, \frac{\sqrt{ } R}{\sqrt{ } \Delta}, \mathbb{D}, \sqrt{ } \Theta\right)
$$

Likewise, the Killing vector field $\boldsymbol{\xi}=\partial_{t}$ is given by

$$
\boldsymbol{\xi}=\frac{1}{\sqrt{ } \Sigma}(\sqrt{ } \Delta, 0, a \sin \vartheta, 0)
$$

Since $\boldsymbol{K}$ is both parallel propagated along $\gamma$ and null, we may apply Lemma 3.1 to obtain a vector field $\boldsymbol{Y}$ that is parallel propagated along $\gamma$. We have

$$
\frac{d}{d s} \beta_{K}=\eta_{a b} K^{a} \xi^{b}=E,
$$

so that $\beta_{\boldsymbol{K}}=E s$, where $s$ is the affine parameter of the null geodesic. We then immediately obtain using (3.8) that the vector field $\boldsymbol{Y}$ defined in the symmetric frame by

$$
\begin{array}{r}
\boldsymbol{Y}=\frac{1}{\sqrt{\kappa \Sigma}}\left(\frac{E s \mathbb{P}-r \sqrt{ } R}{\sqrt{ } \Delta}, \frac{E s \sqrt{ } R-r \mathbb{P}}{\sqrt{ } \Delta},\right. \\
E s \mathbb{D}+a \cos \vartheta \sqrt{ } \Theta, E s \sqrt{ } \Theta-a \cos \vartheta \mathbb{D}),
\end{array}
$$


is parallel propagated along $\gamma$. We now apply Lemma (3.1) to the vector field $Y$ and obtain an additional vector field $\boldsymbol{X}$ that is parallel propagated along $\gamma$. We have

$$
\frac{d}{d s} \beta_{\boldsymbol{Y}}=\eta_{a b} Y^{a} \xi^{b}=\frac{E^{2} s-r \dot{r}-a^{2} \cos \vartheta \sin \vartheta \dot{\vartheta}}{\sqrt{ } \kappa},
$$

where the dot denotes differentiation with respect to the affine parameter $s$. Whence,

$$
\beta_{\boldsymbol{Y}}=\frac{E^{2} s^{2}-r^{2}+a^{2} \cos ^{2} \vartheta}{2 \sqrt{ } \kappa} .
$$

We conclude then that

$$
\begin{aligned}
\boldsymbol{X}=\frac{1}{\kappa \sqrt{ } \Sigma}\left(\frac{\mathbb{P} \beta_{+}-r E s \sqrt{ } R}{\sqrt{ } \Delta}, \frac{\sqrt{ } R \beta_{+}-r E s \mathbb{P}}{\sqrt{ } \Delta},\right. \\
\left.\mathbb{D} \beta_{-}+a \cos \vartheta E s \sqrt{ } \Theta, \sqrt{ } \Theta \beta_{-}-a \cos \vartheta E s \mathbb{D}\right),
\end{aligned}
$$

where

$$
2 \beta_{ \pm}:=E^{2} s^{2} \pm \Sigma
$$

is parallel propagated along $\gamma$.

Note that $\eta_{a b} X^{a} K^{b}=1$ so that $\boldsymbol{X}$ and $\boldsymbol{K}$ are not orthogonal. Thus, we cannot apply Lemma 3.1 to construct a fourth vector field that is parallel propagated along $\gamma$. However, we can use the Killing-Yano tensor $\boldsymbol{f}$ directly to obtain another vector that is parallel propagated along the affinely parametrized null geodesic $\gamma$ with tangent vector $\boldsymbol{K}$. Indeed, it follows immediately from the Killing-Yano equation (2.20) that the vector field $\boldsymbol{Z}$ defined by

$$
Z^{a}=f_{b}^{a} K^{b},
$$

is parallel propagated along $\gamma$. We are of course free to scale $\boldsymbol{Z}$ by any constant, and we will choose this constant to be equal to $\frac{1}{\sqrt{\kappa}}$ so as to simplify the orthogonality relations between the vector fields comprising the parallel propagated frame. Applying (3.18) and scaling $\boldsymbol{Z}$ as above, we obtain

$$
\boldsymbol{Z}=\frac{1}{\sqrt{\kappa \Sigma}}\left(\frac{a \cos \vartheta \sqrt{ } R}{\sqrt{ } \Delta}, \frac{a \cos \vartheta \mathbb{P}}{\sqrt{ } \Delta}, r \sqrt{ } \Theta,-r \mathbb{D}\right)
$$

We thus have a frame $\{\boldsymbol{K}, \boldsymbol{X}, \boldsymbol{Y}, \boldsymbol{Z}\}$ that is parallel propagated along the affinely parametrized null geodesic $\gamma$ with tangent vector $\boldsymbol{K}$. The matrix of 
scalar products for the elements of this frame is given by

$$
\left[\begin{array}{llll} 
& 1 & & \\
1 & & & \\
& & -1 & \\
& & & -1
\end{array}\right] \text {. }
$$

The polarization vector $\digamma$ is orthogonal to $\boldsymbol{K}$. Since $\boldsymbol{K}$ is null and parallel propagated along itself, $\digamma$ is only determined modulo $\boldsymbol{K}$. That is, if $\digamma$ satisfies (3.1) and (3.2), then so does

$$
\digamma^{\prime}=\digamma+c \boldsymbol{K}
$$

where $c$ is a real constant.

Definition 3.2 (2-Plane of Polarization along $\gamma$ ). We choose initial conditions such that $\boldsymbol{\digamma}_{\gamma(0)} \in \operatorname{span}\left\{\boldsymbol{Y}_{\gamma(0)}, \boldsymbol{Z}_{\gamma(0)}\right\}$. Then, $\boldsymbol{\digamma}_{\gamma(s)} \in \operatorname{span}\left\{\boldsymbol{Y}_{\gamma(s)}\right.$, $\left.\boldsymbol{Z}_{\gamma(s)}\right\}$ for all $s$, since $\boldsymbol{\digamma}$ has constant components in $\{\boldsymbol{K}, \boldsymbol{X}, \boldsymbol{Y}, \boldsymbol{Z}\}$. This defines the 2-plane of polarization

$$
\mathcal{P}_{\gamma(s)}:=\operatorname{span}\left\{\boldsymbol{Y}_{\gamma(s)}, \boldsymbol{Z}_{\gamma(s)}\right\} \subset\langle\boldsymbol{K}\rangle^{\perp}
$$

at each event $\gamma(s) \in M$.

In order to simplify the computation we define an orthonormal frame that is parallel propagated along the null geodesic $\left(\boldsymbol{L}_{(0)}, \boldsymbol{L}_{(1)}, \boldsymbol{L}_{(2)}, \boldsymbol{L}_{(3)}\right)$ by a constant coefficient transformation of $\{\boldsymbol{K}, \boldsymbol{X}, \boldsymbol{Y}, \boldsymbol{Z}\}$ as follows.

$$
\boldsymbol{L}_{(0)}:=\frac{1}{\sqrt{2}}(\boldsymbol{K}+\boldsymbol{X}), \boldsymbol{L}_{(1)}:=\frac{1}{\sqrt{2}}(\boldsymbol{K}-\boldsymbol{X}), \boldsymbol{L}_{(2)}:=\boldsymbol{Y}, \boldsymbol{L}_{(3)}:=\boldsymbol{Z}
$$

We note here the explicit expressions for the elements of frame $\boldsymbol{L}_{(a)}$ with respect to the symmetric frame.

$$
\begin{aligned}
\boldsymbol{L}_{(0)}=\frac{1}{\kappa \sqrt{2 \Sigma}}\left[\frac{\mathbb{P}\left(\kappa+\beta_{+}\right)-E s \sqrt{ } R}{\sqrt{ } \Delta}, \frac{\sqrt{ } R\left(\kappa+\beta_{+}\right)-E s \mathbb{P}}{\sqrt{ } \Delta},\right. \\
\left.\mathbb{D}\left(\kappa+\beta_{-}\right)+a \cos \vartheta E s \sqrt{ } \Theta, \sqrt{ } \Theta\left(\kappa+\beta_{-}\right)-a \cos \vartheta E s \mathbb{D}\right],
\end{aligned}
$$




$$
\begin{aligned}
\boldsymbol{L}_{(1)}= & \frac{1}{\kappa \sqrt{2 \Sigma}}\left[\frac{\mathbb{P}\left(\kappa-\beta_{+}\right)+E s \sqrt{ } R}{\sqrt{ } \Delta}, \frac{\sqrt{ } R\left(\kappa-\beta_{+}\right)+E s \mathbb{P}}{\sqrt{ } \Delta},\right. \\
& \left.\mathbb{D}\left(\kappa-\beta_{-}\right)-a \cos \vartheta E s \sqrt{ } \Theta, \sqrt{ } \Theta\left(\kappa-\beta_{-}\right)+a \cos \vartheta E s \mathbb{D}\right], \\
\boldsymbol{L}_{(2)}= & \frac{1}{\sqrt{\kappa \Sigma}}\left[\frac{E s \mathbb{P}-r \sqrt{ } R}{\sqrt{ } \Delta}, \frac{E s \sqrt{ } R-r \mathbb{P}}{\sqrt{ } \Delta},\right. \\
\boldsymbol{L}_{(3)}= & \frac{1}{\sqrt{\kappa \Sigma}}\left[\frac{a \cos \vartheta \sqrt{ } R}{\sqrt{ } \Delta}, \frac{a \cos \vartheta \mathbb{P}}{\sqrt{ } \Delta}, r \sqrt{ } \Theta,-r \mathbb{D}\right] .
\end{aligned}
$$

\section{Defining and measuring Faraday rotation}

In order to define the Faraday rotation we need to pin down the class of observers who are involved in the communication protocol and specify the frames with respect to which they are measuring the polarization. We have already seen that Carter's symmetric frame frame is closely tied to intrinsic geometric properties of the Kerr metric. This makes it an ideal candidate for formulating the Faraday rotation in a geometrically meaningful fashion.

In the definition of Carter's null frame, which is dual to the co-frame defined in (2.14)-(2.17), the arbitrary scaling of the vectors $\boldsymbol{\ell}$ and $\boldsymbol{n}$ has been fixed by the action of the involution. Thus, one has a natural time-like vector field $\boldsymbol{U}$, namely

$$
\boldsymbol{U}:=\frac{1}{\sqrt{2}}(\boldsymbol{\ell}+\boldsymbol{n})=\frac{1}{\sqrt{\Sigma \Delta}}\left(\left(r^{2}+a^{2}\right) \frac{\partial}{\partial t}+a \frac{\partial}{\partial \varphi}\right)
$$

where $\boldsymbol{\ell}$ and $\boldsymbol{n}$ are given by (2.3) and (2.4). This identifies a family of observers whose 4 -velocities are a symmetric linear combination of the principal null directions $\boldsymbol{\ell}$ and $\boldsymbol{n}$. We call them Carter observers. We choose to work with Carter observers because it follows from the discussion of Section 2 that they are defined geometrically in terms of the principal null directions of the Weyl tensor and the involution $L$. They exist everywhere outside the event horizon including the region between the event horizon and the stationary limit surface $r=r_{s}$ where the Killing vector field $\boldsymbol{\xi}=\partial_{t}$ becomes null. Their coordinate angular velocity is $a /\left(r^{2}+a^{2}\right)$, which is exactly the coordinate angular velocity of the event horizon with $r=r_{+}$. Therefore, this class of observers is uniquely suited to analyze the behaviour of test particles near the horizon. 
We choose the observers' frames to be duals of the symmetric coframe defined in equations (2.14)-(2.17). We shall see that since the symmetric frame is so well adapted to the geometry of Kerr spacetime, this choice will greatly simplify the computation and allow us to obtain a compact, closed form expression for the geometrically induced Faraday rotation of the polarization vector.

We decompose the tangent space $T_{x} M$ at any event $x$ along the worldline of the observer with 4-velocity $\boldsymbol{U}$ into an orthogonal direct sum of spacelike and timelike vector spaces in accordance with the observer's decomposition of spacetime by projecting vectors onto the observer's frame at event $x$. That is,

$$
T_{x} M=\langle\boldsymbol{U}\rangle \oplus \Sigma_{3},
$$

where $\Sigma_{3}:=\langle\boldsymbol{U}\rangle^{\perp}$. In what follows, we shall supress the label $x$ for the event with the understanding that this $1+3$ decomposition is only valid at a given event. The observer's frame defines a projection map $\pi: T_{x} M \longrightarrow \Sigma_{3}$,

$$
\pi(\boldsymbol{X})=: \overrightarrow{\boldsymbol{X}}=\left[\begin{array}{c}
X^{1} \\
X^{2} \\
X^{3}
\end{array}\right]
$$

Given the direction 3-vector of the photon $\pi(\boldsymbol{K})=\overrightarrow{\boldsymbol{K}}$, consider the 2-plane $\langle\overrightarrow{\boldsymbol{K}}\rangle^{\perp} \subset \Sigma_{3}$ passing through the origin and orthogonal to it. Given a pair of orthonormal basis vectors for this 2-plane, $\left\{\overrightarrow{\boldsymbol{b}_{1}}, \overrightarrow{\boldsymbol{b}_{2}}\right\}$, we can write a general polarization 3-vector as

$$
\vec{\digamma}=c_{1} \overrightarrow{\boldsymbol{b}_{1}}+c_{2} \overrightarrow{\boldsymbol{b}_{2}}
$$

for real constants $c_{1}$ and $c_{2}$. It is crucial this choice of basis vectors not be made arbitrarily. We choose basis vectors on intrinsic geometric criteria, which are independent of coordinate descriptions. First, we project the principal null direction $\ell$ onto $\Sigma_{3}$ and obtain the corresponding 3 -vector $\pi(\ell):=\vec{\ell}$ using the prescription (4.3). Then, we set the basis vectors in the plane of polarization to be

$$
\overrightarrow{b_{1}}:=\frac{\vec{\ell} \times \vec{K}}{\|\vec{\ell} \times \vec{K}\|},
$$




$$
\overrightarrow{\boldsymbol{b}_{2}}:=\frac{\overrightarrow{\boldsymbol{K}} \times \overrightarrow{\boldsymbol{b}_{1}}}{\left\|\overrightarrow{\boldsymbol{K}} \times \overrightarrow{\boldsymbol{b}_{1}}\right\|} .
$$

We are finally ready to spell out the communication protocol. Let Alice and Bob be two Carter observers in the Kerr exterior. In order to communicate with Bob, Alice sends a polarized photon along a null geodesic $\gamma(s)$ that intersects with Bob's worldline. Alice polarizes the photon in the basis (4.5)(4.6) at the event $x_{A}$, which we denote here by $\vec{\digamma}_{\text {in. }}$ When Bob sees the photon he also measures its polarization by projecting it onto the basis (4.5)(4.6) at the event $x_{B}$ to obtain $\vec{\digamma}$ out. Note that since these bases are defined intrinsically they can agree in advance on the choice of these bases and set them up locally without further communication once they have embarked on their orbits.

The geometrically induced Faraday rotation of the polarization vector of a photon as it transverses the Kerr exterior from Alice and Bob is then given by the angle $\chi$ such that

$$
\vec{\digamma}_{\text {out }}:=\left[\begin{array}{cc}
\cos \chi & -\sin \chi \\
\sin \chi & \cos \chi
\end{array}\right] \vec{\digamma}_{\text {in }} .
$$

Remark 4.1. The reference frame on which the measurement of Faraday rotation depends so critically is not the orthonormal symmetric frame. Rather, it is the measurement basis $\left\{\overrightarrow{\boldsymbol{b}_{1}}, \overrightarrow{\boldsymbol{b}_{2}}\right\}$ which plays the role of the reference frame.

Remark 4.2. Using the intrinsic geometry of Kerr to solve the problem of choosing a set of basis vectors for the plane of polarization as specified in this section simultaneously solves the problem of sharing frames and minimizes the informational requirement on the observers. Note that such a strategy is simply unavailable in Minkowski spacetime where no direction is similarly privileged: there is too much symmetry. ${ }^{2}$ In our case, the type D character of Kerr geometry provides just enough symmetry to allow for the possibility of the present protocol with its minimal communication requirements.

We are now in a position to prove the following proposition.

Proposition 4.3. Consider observers confined to the equatorial plane Eq $:=$ $\left\{-\infty<t<+\infty, r_{+}>r>+\infty, \vartheta=\pi / 2,0 \leq \varphi<2 \pi\right\}$. There is no Faraday rotation for photons confined to the equatorial plane of Kerr geometry.

\footnotetext{
${ }^{2}$ Of course, in Minkowski space one can use alternative protocols.
} 
Proof. Consider the vector field $\boldsymbol{V}:=-\frac{1}{r} \partial_{\vartheta}$. This is unit norm vector field which is orthogonal to the equatorial plane when restricted to it. We will by an abuse of notation use $\boldsymbol{V}$ to denoted $\left.\boldsymbol{V}\right|_{E q}$. An easy calculation shows that $\pi(\boldsymbol{V})=\overrightarrow{\boldsymbol{b}_{1}}$. Using the expressions for the Christoffel symbols given in Appendix A, we obtain

$$
\nabla_{\boldsymbol{K}} \boldsymbol{V}=0
$$

By $(3.1)$ and (3.2), it follows that $\digamma^{a} V^{b} \eta_{a b}=0$, which together with $\pi(\boldsymbol{V})=$ $\overrightarrow{\boldsymbol{b}_{1}}$ implies

$$
\vec{\digamma} \cdot \overrightarrow{b_{1}}=\text { constant }
$$

Corollary 4.4. There is no Faraday rotation in the Schwarzschild geometry.

Proof. Since the Schwarzschild geometry is spherically symmetric, geodesics are confined to planes through the origin [8]. Therefore, the exact same argument as we used for the equatorial plane in Kerr can be used here. Any plane through the origin can be viewed as the equatorial plane of a degenerate Kerr solution with $a=0$.

Proposition 4.5. There is no Faraday rotation for orbits confined to the axis of symmetry of Kerr geometry.

Proof. The unit vector $\frac{\sqrt{ } \Delta}{\sqrt{ } \Sigma} \partial_{r}$ plays the same role as $\boldsymbol{V}$ in Proposition 4.3. The proof follows the same argument as Proposition 4.3 and is therefore omitted.

Remark 4.6. We conjecture that the vanishing of the Faraday rotation characterizes all totally geodesic submanifolds of Kerr geometry.

\section{Exact, closed form expression for the Faraday rotation in Kerr geometry}

The direction 3 -vector corresponding to the principal null direction $\ell$ in the symmetric frame is given in $\Sigma_{3}$ by 


$$
\overrightarrow{\boldsymbol{\ell}}=\left[\begin{array}{l}
1 \\
0 \\
0
\end{array}\right]
$$

and direction 3 -vector for an arbitrary photon in $\Sigma_{3}$ is given by

$$
\overrightarrow{\boldsymbol{K}}=\frac{1}{\mathbb{P}}\left[\begin{array}{c}
\sqrt{ } R \\
\sqrt{ } \Delta \mathbb{D} \\
\sqrt{\Delta \Theta}
\end{array}\right]
$$

Now, using (4.5)-(4.6), we obtain the following basis for the plane of polarization:

$$
\begin{aligned}
& \overrightarrow{\boldsymbol{b}_{1}}=\frac{1}{\sqrt{ } \kappa}\left[\begin{array}{c}
0 \\
-\sqrt{ } \Theta \\
\mathbb{D}
\end{array}\right], \\
& \overrightarrow{\boldsymbol{b}_{2}}=\frac{1}{\sqrt{ } \kappa \mathbb{P}}\left[\begin{array}{c}
-\kappa \sqrt{ } \Delta \\
\mathbb{D} \sqrt{ } R \\
\sqrt{R \Theta}
\end{array}\right] .
\end{aligned}
$$

We may choose the affine parameter $s$ so that $s=0$ at the event $x_{A}$ where the null geodesic intersects Alice's worldline and $s=s_{*}$ at the event $x_{B}$ where the null geodesic intersects Bob's worldline. The basis vectors $\boldsymbol{Y}_{\gamma(s)}$ and $\boldsymbol{Z}_{\gamma(s)}$ of the plane of polarization $\mathcal{P}_{\gamma(s)} \in T_{\gamma(s)} M$ can now be projected onto the 2-plane of polarization in $\Sigma_{3}$, in the basis (5.3)-(5.4):

$$
\begin{aligned}
& \overrightarrow{\boldsymbol{y}}:=\left[\begin{array}{c}
\pi(\boldsymbol{Y}) \cdot \overrightarrow{\boldsymbol{b}_{1}} \\
\pi(\boldsymbol{Y}) \cdot \overrightarrow{\boldsymbol{b}_{2}}
\end{array}\right]:=\frac{1}{\sqrt{ } \Sigma}\left[\begin{array}{c}
-a \cos \vartheta \\
r
\end{array}\right], \\
& \overrightarrow{\boldsymbol{z}}:=\left[\begin{array}{l}
\pi(\boldsymbol{Z}) \cdot \overrightarrow{\boldsymbol{b}_{1}} \\
\pi(\boldsymbol{Z}) \cdot \overrightarrow{\boldsymbol{b}_{2}}
\end{array}\right]:=\frac{1}{\sqrt{ } \Sigma}\left[\begin{array}{c}
-r \\
-a \cos \vartheta
\end{array}\right] .
\end{aligned}
$$

Note that terms with $s$ do not survive. All the dynamic information is contained in the behaviour of $r$ and $\vartheta$. Note as well that the polarization vector has constant components in (5.5) and (5.6). At the event $x_{A}=\gamma(s=0)$, let Alice choose

$$
\vec{\digamma}_{\text {in }}=\left[\begin{array}{l}
c_{1} \\
c_{2}
\end{array}\right]=c_{1} \overrightarrow{\boldsymbol{b}_{1}}+c_{2} \overrightarrow{\boldsymbol{b}_{2}},
$$


that is,

$$
\vec{\digamma}_{\text {in }}=\frac{1}{\sqrt{ } \Sigma_{0}}\left(\left(r_{0} c_{2}-c_{1} a \cos \vartheta_{0}\right) \overrightarrow{\boldsymbol{y}}-\left(c_{1} r_{0}+c_{2} a \cos \vartheta_{0}\right) \overrightarrow{\boldsymbol{z}}\right) .
$$

The components of $\digamma$, which stay constant with respect to the parallel propagated frame $\boldsymbol{L}_{(a)}$ given by (3.24), are therefore

$$
\digamma^{(a)}=-\frac{1}{\sqrt{ } \Sigma_{0}}\left[\begin{array}{c}
0 \\
0 \\
c_{1} a \cos \vartheta_{0}-c_{2} r_{0} \\
c_{1} r_{0}+c_{2} a \cos \vartheta_{0}
\end{array}\right] .
$$

At $x_{B}=\gamma\left(s=s_{*}\right)$, Bob measures $\vec{\digamma}$ in the basis $\left\{\overrightarrow{\boldsymbol{b}_{1}}, \overrightarrow{\boldsymbol{b}_{2}}\right\}$, to obtain $\vec{\digamma}$ out which is given by (we supress the subscript for $s=s_{*}$ ):

$$
\begin{aligned}
& \vec{\digamma}_{\text {out }}=\frac{1}{\sqrt{ } \Sigma_{0}}\left(\left(r_{0} c_{2}-c_{1} a \cos \vartheta_{0}\right) \overrightarrow{\boldsymbol{y}}(s)-\left(c_{1} r_{0}+c_{2} a \cos \vartheta_{0}\right) \overrightarrow{\boldsymbol{z}}(s)\right) \\
& =\frac{1}{\sqrt{\Sigma_{0} \Sigma}}\left[\begin{array}{l}
c_{1}\left(r(s) r_{0}+a^{2} \cos \vartheta_{0} \cos \vartheta(s)\right)-c_{2}\left(r_{0} a \cos \vartheta(s)-r(s) a \cos \vartheta_{0}\right) \\
c_{1}\left(r_{0} a \cos \vartheta(s)-r(s) a \cos \vartheta_{0}\right)+c_{2}\left(r(s) r_{0}+a^{2} \cos \vartheta_{0} \cos \vartheta(s)\right)
\end{array}\right] \\
& =\frac{1}{\sqrt{\Sigma_{0} \Sigma}}\left[\begin{array}{cc}
\left(r(s) r_{0}+a^{2} \cos \vartheta_{0} \cos \vartheta(s)\right) & -\left(r_{0} a \cos \vartheta(s)-r(s) a \cos \vartheta_{0}\right) \\
\left(r_{0} a \cos \vartheta(s)-r(s) a \cos \vartheta_{0}\right) & \left(r(s) r_{0}+a^{2} \cos \vartheta_{0} \cos \vartheta(s)\right)
\end{array}\right] \vec{\digamma}_{\text {in }} .
\end{aligned}
$$

The rotation matrix in $(5.12)$ is therefore

$$
\frac{1}{\sqrt{\Sigma_{0} \Sigma}}\left[\begin{array}{cc}
\left(r(s) r_{0}+a^{2} \cos \vartheta_{0} \cos \vartheta(s)\right) & -\left(r_{0} a \cos \vartheta(s)-r(s) a \cos \vartheta_{0}\right) \\
\left(r_{0} a \cos \vartheta(s)-r(s) a \cos \vartheta_{0}\right) & \left(r(s) r_{0}+a^{2} \cos \vartheta_{0} \cos \vartheta(s)\right)
\end{array}\right] .
$$

This implies that the Faraday rotation is given by the angle $\chi$ such that

$$
\tan \chi(s)=\frac{a\left(r(s) \cos \vartheta_{0}-r_{0} \cos \vartheta(s)\right)}{r(s) r_{0}+a^{2} \cos \vartheta_{0} \cos \vartheta(s)} .
$$

\section{Summary and discussion}

The radical simplicity of (5.12) stems from our geometrically motivated choice of observers, frames, polarization plane and measurement basis. Exploiting the existence of the Killing-Yano tensor in Kerr geometry, we were able to obtain a parallel propagated frame, thereby transforming the problem of parallel transport of the polarization vector into one of raising and 
lowering frame indices. The fact that the parallel propagated frame provides two vector fields that form a natural basis for the plane of polarization in $T_{x} M$ at each point $x \in \gamma(s)$ reduces the calculation of Faraday rotation to an elementary computation.

Choosing a specific class of observers in order to make it easy to compute the result does not limit the applicability of the technique to just those observers. Since the components in the parallel propagated frame have to stay constant, in order to determine the Faraday rotation measured by another choice of observers, we must apply local Lorentz transformations only at the two events $x_{A}$ and $x_{B}$ in order to relate the frames of the arbitrary observers to the frames of the Carter observers going through the same spacetime events. This is a local transformation, quite distinct from the geometric effect of the Kerr black hole which is a global phenomenon. The analogous question in Minkowski geometry is the study of Wigner rotation which has been extensively analyzed in the massless case $[1,14,24]$.

In order to qualitatively analyze the expression we have obtained for Faraday rotation we present some plots in Appendix B. In each of the 3 tables, the first figure (a) shows the orbital behaviour of the null geodesic with $(r(s), \varphi(s))$ as polar coordinates, the second figure (b) depicts the same orbits in three dimensions with spherical coordinates $(r(s), \vartheta(s), \varphi(s))$, and the last figure (c) depicts the Faraday rotation as a function of the affine parameter $s$.

Tables 1 and 2 show co-rotating orbits since $\Phi>0$, while Table 3 shows a counter-rotating orbit $(\Phi<0)$. The apparent axial symmetry of the Faraday rotation in Tables 1 and 3 is an artifact of our choice of initial data, and not due to their co- and counter-rotating character. In the first two orbits $\dot{\chi}(0)<0$, while for the third one $\dot{\chi}(0)>0$. The sign of $\dot{\chi}(0)$ is determined by the sign of the left hand side of equation (6.1) evaluated at $s=0$.

The set of figures in Table 2 corresponds to an interesting null orbit. A segment of this orbit lies inside the ergosphere (the dotted line in figure (a)), which, somewhat surprisingly, does not seem to have a qualitative effect on the Faraday rotation. Photons on this orbit circumnavigate the black hole before escaping to infinity. That is, the acquired azimuthal angle $\Delta \varphi$ is greater than $2 \pi$. This is possibly why $\chi$ has three critical points for this orbit. However, this conjecture cannot be resolved without a classification of the critical points of $\chi$, which are implicity given by

$$
\frac{r}{\sqrt{ } R}+\frac{\cot \vartheta}{\sqrt{ } \Theta}=0,
$$


where $R$ and $\Theta$ are given by (2.28) and (2.29) respectively. This is a trancendental equation with two elliptic functions with different periods. We are unaware of any methods to obtain explicit solutions.

Finally, we note that the measured Faraday rotation $\chi$ is invariant under the involution $L$ given by (2.8).

The investigations of the present paper suggest a number of avenues of further investigation. First, and perhaps the most pressing, is to study quantum evolution in Kerr geometry and understand how the density matrices describing states evolve as quantum systems are exchanged between observers. Here one is interested in the evolution of wave packets, and not just in the purely geometric problem of propagating polarization vectors along a null geodesic. Recent progress in the Cauchy problem for the Dirac equation in Kerr geometry [11-13] make it possible to study this problem rigorously for Dirac particles. The analogous problem for vector particles would require new advances in our understanding of the Maxwell equations in Kerr geometry.

In quantum information theory a central concern is coping with noise. In order to understand the effect of noise it would be interesting to investigate the sensitivity of our results to perturbations of the initial data.

Finally, we have considered the question of sharing very special frames percisely using geometric features. It would be very interesting to understand how to share more general classes of frames; this is a topic that would involve group theory as well as geometry and would make an appealing complement to the quantum information theory studies of Bartlett et al. [2, 3].

\section{Acknowledgements}

This research was supported by grants from NSERC and by a BRC grant from the Office of Naval Research (N00001 1408 11249). We are very grateful to Eva Hackman, without whose help we would not have been able to plot the geodesics. Farooqui and Panangaden would also like to acknowledge useful conversations with Paul Alsing. 


\section{Appendix A. Christoffel symbols}

The Christoffel symbols are defined by

$$
\Gamma_{j k}^{i}=\frac{1}{2} g^{i l}\left(g_{l j, k}+g_{l k, j}-g_{j k, l}\right)
$$

In Boyer-Lindquist coordinates, the nonzero ones are:

$$
\begin{aligned}
& \Gamma_{r t}^{t}=M\left(r^{2}+a^{2}\right)\left(r^{2}-a^{2} \cos ^{2} \vartheta\right) / \Sigma^{2} \Delta \\
& \Gamma_{\vartheta t}^{t}=-2 M r a^{2} \cos \vartheta \sin \vartheta / \Sigma^{2} \\
& \Gamma_{r \varphi}^{t}=a M \sin ^{2} \vartheta\left(a^{4} \cos ^{2} \vartheta-r^{2} a^{2} \cos ^{2} \vartheta-r^{2} a^{2}-3 r^{4}\right) / \Sigma^{2} \Delta \\
& \Gamma_{\vartheta \varphi}^{t}=2 M r a^{3} \sin ^{3} \vartheta \cos \vartheta / \Sigma^{2} \\
& \Gamma_{t t}^{r}=M\left(r^{2}-a^{2} \cos ^{2} \vartheta\right) \Delta / \Sigma^{3} \\
& \Gamma_{\varphi t}^{r}=-a M \sin ^{2} \vartheta\left(r^{2}-a^{2} \cos ^{2} \vartheta\right) \Delta / \Sigma^{3} \\
& \Gamma_{r r}^{r}=\left(r a^{2} \sin ^{2} \vartheta-M\left(r^{2}-a^{2} \cos ^{2} \vartheta\right)\right) / \Sigma \Delta \\
& \Gamma_{\vartheta r}^{r}=-a^{2} \cos \vartheta \sin \vartheta / \Sigma \\
& \Gamma_{\vartheta \vartheta}^{r}=-r \Delta / \Sigma \\
& \Gamma_{\varphi \varphi}^{r}=\Delta \sin ^{2} \vartheta\left(M a^{2} \sin ^{2} \vartheta\left(r^{2}-a^{2} \cos ^{2} \vartheta\right)-r \Sigma^{2}\right) / \Sigma^{3} \\
& \Gamma_{t t}^{\vartheta}=-2 M r a^{2} \sin \vartheta \cos \vartheta / \Sigma^{3} \\
& \Gamma_{\varphi t}^{\vartheta}=2 M r a \sin \vartheta \cos \vartheta\left(r^{2}+a^{2}\right) / \Sigma^{3} \\
& \Gamma_{r r}^{\vartheta}=a^{2} \sin \vartheta \cos \vartheta / \Sigma \Delta \\
& \Gamma_{r \vartheta}^{\vartheta}=r / \Sigma \\
& \Gamma_{\vartheta \vartheta}^{\vartheta}=-a^{2} \sin \vartheta \cos \vartheta / \Sigma \\
& \Gamma_{\varphi \varphi}^{\vartheta}=-\cos \vartheta \sin \vartheta\left(\Sigma^{2} \Delta+2 M r\left(r^{4}+a^{2}+2 r^{2}\right)\right) / \Sigma^{3} \\
& \Gamma_{r t}^{\varphi}=M a\left(r^{2}-a^{2} \cos ^{2} \vartheta\right) / \Sigma^{2} \Delta \\
& \Gamma_{\vartheta t}^{\varphi}=-2 M r a \cot \vartheta / \Sigma^{2} \\
& \Gamma_{r \varphi}^{\varphi}=\left((r-M) \Sigma^{2}-M\left(r^{2}+a^{2}\right)\left(r^{2}-a^{2} \cos ^{2} \vartheta\right)\right) / \Sigma^{2} \Delta \\
& \Gamma_{\vartheta \varphi}^{\varphi}=\cot \vartheta+2 M r a^{2} \cos \vartheta \sin \vartheta / \Sigma^{2}
\end{aligned}
$$




\section{Appendix B. Plots}

Table B1: A co-rotating orbit with $\Phi=3, \kappa=12$ and initial data $r(0)=$ $20, \vartheta(0)=1.57$, and $\varphi(0)=0$.

(a) The orbit in polar coordinates $(x=r \cos \varphi, y=r \sin \varphi)$.

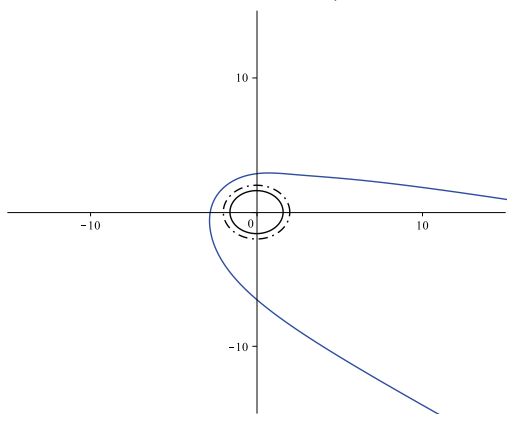

(b) The orbit in 3D spherical coordinates $(x=r \cos \varphi \sin \vartheta, y=r \sin \varphi \sin \vartheta, z=r \cos \vartheta)$.

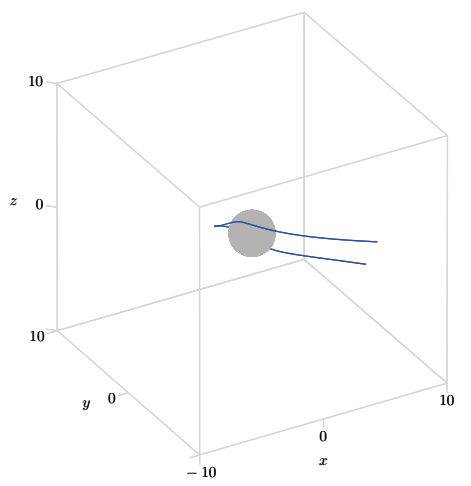

(c) The Faraday rotation angle as a function of the affine parameter $s$

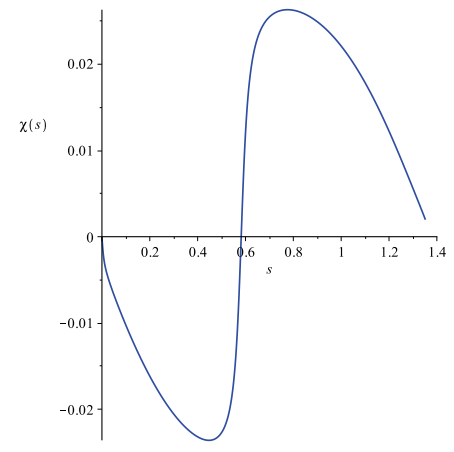


Table B2: A co-rotating orbit with $\Phi=3.11, \kappa=6.97$ and initial data $r(0)=20, \vartheta(0)=1.57$, and $\varphi(0)=0$.

(a) The orbit in polar coordinates $(x=r \cos \varphi, y=r \sin \varphi)$.

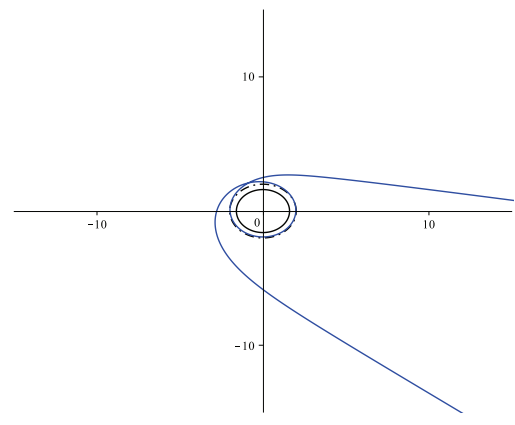

(b) The orbit in $3 \mathrm{D}$ spherical coordinates $(x=r \cos \varphi \sin \vartheta, y=r \sin \varphi \sin \vartheta, z=r \cos \vartheta)$.

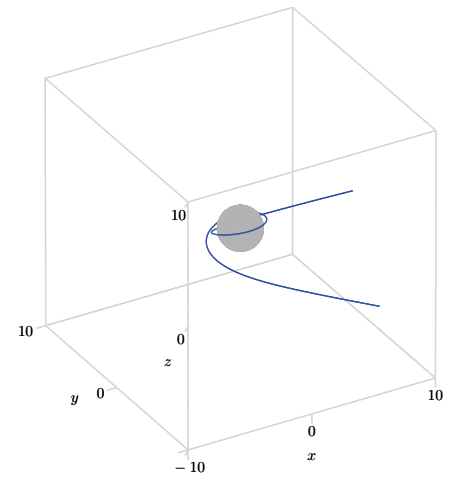

(c) The Faraday rotation angle as a function of the affine parameter $s$

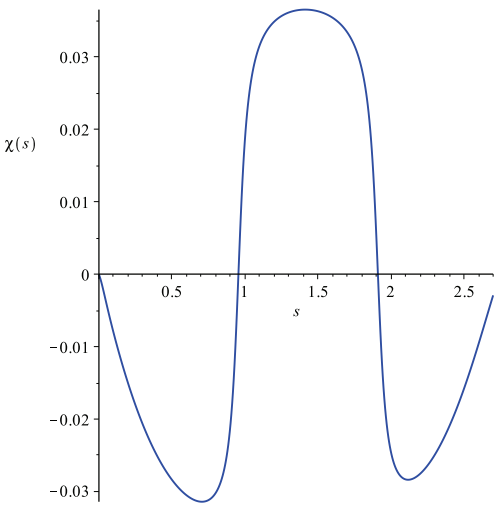


Table B3: A counter-rotating orbit with $\Phi=-6, \kappa=60$ and initial data $r(0)=20, \vartheta(0)=1.57$, and $\varphi(0)=0$.

(a) The orbit in polar coordinates $(x=r \cos \varphi, y=r \sin \varphi)$.

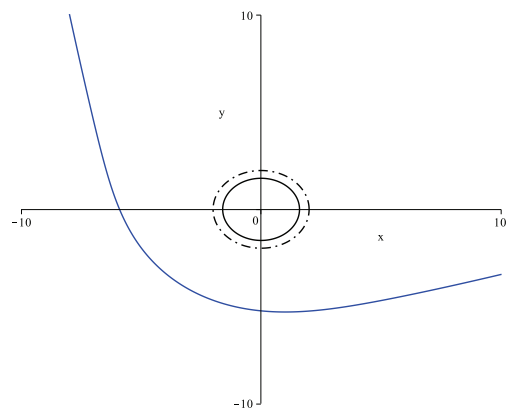

(b) The orbit in 3D spherical coordinates $(x=r \cos \varphi \sin \vartheta, y=r \sin \varphi \sin \vartheta, z=r \cos \vartheta)$.

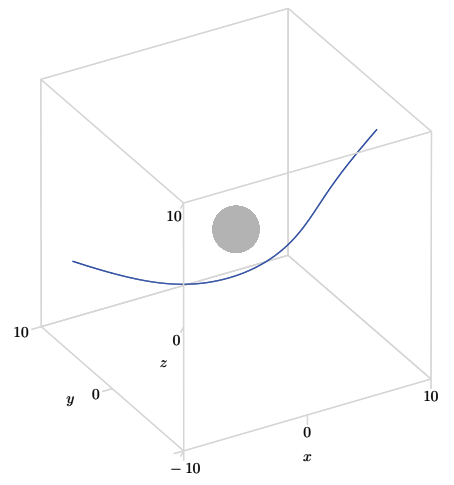

(c) The Faraday rotation angle as a function of the affine parameter $s$

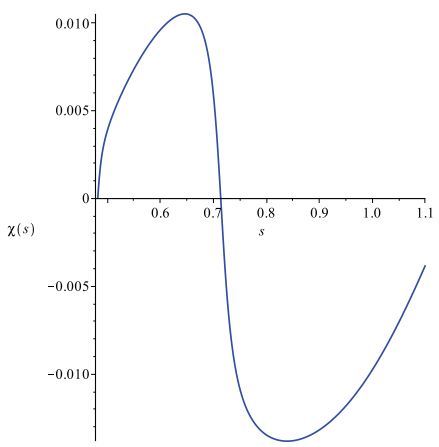




\section{References}

[1] Paul M Alsing and Gerard J Milburn. Lorentz invariance of entanglement. preprint, 2002. arXiv:quant-ph/0203051.

[2] Stephen D. Bartlett, Terry Rudolph and Robert W. Spekkens. Classical and quantum communication without a shared reference frame. Phys. Rev. Lett., 91:027901, Jul 2003.

[3] Stephen D. Bartlett, Terry Rudolph and Robert W. Spekkens. Reference frames, superselection rules and quantum information. Rev. Mod. Phys., 79:555-609, Apr 2007.

[4] Aharon Brodutch, Tommaso F. Demarie and Daniel R. Terno. Photon polarization and geometric phase in general relativity. Physical Review D, 84(10):104043, 2011.

[5] Brandon Carter. Global structure of the Kerr family of gravitational fields. Physical Review, 174(5):1559, 1968.

[6] Brandon Carter. Hamilton-Jacobi and Schrodinger separable solutions of Einstein's equations. Comm. Math. Phys., 10:280-310, 1968.

[7] Brandon Carter. Killing horizons and orthogonally transitive groups in space-time. Journal of Mathematical Physics, 10:70, 1969.

[8] Subrahmanyan Chandrasekhar. The Mathematical Theory of Black Holes. Number 69 in International Series of Monographs on Physics. Oxford University Press, 2nd edition edition, 1992. 1st edition, published 1983.

[9] Robert Debever, Raymond G. McLenaghan and Nessim Tariq. Riemannian-maxwellian invertible structures in general relativity. General Relativity and Gravitation, 10(10):853-879, 1979.

[10] Francisco Fayos and Josep Llosa. Gravitational effects on the polarization plane. General Relativity and Gravitation, 14(10):865-877, 1982.

[11] Felix Finster, Niky Kamran, Joel Smoller and Shing-Tung Yau. Decay rates and probability estimates for massive Dirac particles in the Kerr-Newman black hole geometry. Communications in Mathematical Physics, 230(2):201-244, 2002. 
[12] Felix Finster, Niky Kamran, Joel Smoller and Shing-Tung Yau. The long-time dynamics of Dirac particles in the Kerr-Newman black hole geometry. Advances in Theoretical and Mathematical Physics, 7(1):2552, 2003.

[13] Felix Finster, Niky Kamran, Joel Smoller and Shing-Tung Yau. Linear waves in the Kerr geometry: a mathematical voyage to black hole physics. Bulletin of the American Mathematical Society, 46(4):635-659, 2009.

[14] Robert M Gingrich, Bergou, J Attila and Christoph Adami. Entangled light in moving frames. Physical Review A, 68(4):042102, 2003.

[15] Brendan B. Godfrey. Mach's principle, the Kerr metric, and black-hole physics. Physical Review D, 1(10):2721, 1970.

[16] Hideki Ishihara, Masaaki Takahashi and Akira Tomimatsu. Gravitational Faraday rotation induced by a Kerr black hole. Phys. Rev. D, $\mathbf{3 8}(2): 472,1988$.

[17] David Kubiznak, Valeri P. Frolov, Pavel Krtous and Patrick Connell. Parallel-propagated frame along null geodesics in higher-dimensional black hole spacetimes. Phys. Rev. D, 79:024018, Jan 2009.

[18] Jean-Alain Marck. Parallel-tetrad on null geodesics in Kerr-Newman space-time. Physics Letters A, 97(4):140-142, 1983.

[19] Michael Nielsen and Issac Chuang. Quantum Computation and Quantum Information. Cambridge University Press, 2000.

[20] Mohammad Nouri-Zonoz. Gravitoelectromagnetic approach to the gravitational Faraday rotation in stationary spacetimes. Physical Review D, 60(2):024013, 1999.

[21] Jerzy Plebanski. Electromagnetic waves in gravitational fields. Physical Review, 118(5):1396, 1960.

[22] Mauro Sereno. Gravitational Faraday rotation in a weak gravitational field. Phys. Rev. D, 69:087501, Apr 2004.

[23] George V. Skrotskii. On the influence of gravity on the light propagation. Akademiia Nauk SSSR Doklady, 114:73-76, 1957.

[24] Hiroaki Terashima and Masahito Ueda. Einstein-podolsky-rosen correlation seen from moving observers. preprint, 2002. arXiv:quant-ph/ 0204138. 
Department of Mathematics and Statistics, McGill University 805 Sherbrooke Street West, Montreal QC H3A 0B9, Canada E-mail address: farooqui@math.mcgill.ca

Department of Mathematics and Statistics, McGill University 805 Sherbrooke Street West, Montreal QC H3A 0B9, Canada E-mail address: nkamran@math.mcgill.ca

School of Computer Science, McGill University 3480 University Street, Montreal QC H3A 0E9, Canada E-mail address: prakash@cs.mcgill.ca 